\title{
Early Estimation of the Basic Reproduction Number Using Minimal Outbreak Data
}

\author{
Carl Grafe* \\ ${ }^{1}$ Salt Lake County Health Department, Salt Lake City, UT, USA; ${ }^{2}$ University of Utah, Salt Lake City, UT, USA
}

\section{Objective}

To present a modification to an established approach to estimating the basic reproduction number to allow estimates to be obtained at any point during an outbreak using only the current case count, number currently ill, and the size of the at-risk population.

\section{Introduction}

The basic reproduction number represents the number of secondary infections expected to be caused by an infectious individual introduced into an entirely susceptible population. ${ }^{1}$ It is a fundamental measure used to characterize infectious disease outbreaks and is essential in developing mathematical models to determine appropriate interventions. ${ }^{2}$ Much work has been done to investigate methods for estimating the basic reproduction number during the early stages of infectious disease outbreaks. ${ }^{3}$ However, these methods often require data that may not be readily available at the beginning of an outbreak. ${ }^{3}$ An approach developed by Becker ${ }^{4}$ has been widely used to estimate the basic reproduction number using only the final case count and size of the at-risk population. ${ }^{5}$ A modification to this approach is proposed that allows estimates to be obtained earlier in an outbreak using only the current case count, number currently ill, and the size of the at-risk population.

\section{Methods}

The formula derived by Becker includes the number of infected subjects who have subsequently recovered, which is assumed to be known only after the outbreak has concluded. However, the number who are currently ill may also feasibly be known during an outbreak. Simply subtracting this number from the current case count yields an estimate of the current number who have recovered, allowing the formula to be applied in the same manner as designed, only using earlier data.

A stochastic SIR (susceptible, infectious, removed) compartmental mathematical model was developed in order to test the performance of the original approach compared with the proposed modification to the approach. The model was run 1,000 times at each of nine assumed basic reproduction numbers from 1 to 5 at 0.5 intervals, which were randomly sampled from a gamma distribution throughout each outbreak in order to simulate individual variation. The duration of illness was generically allowed to vary between 1 and 5 days. The standard approach was applied after the conclusion of each outbreak, and the modified approach was applied at a randomly selected point between the beginning and end of each outbreak. The mean of these estimates was then taken to obtain an estimate for each selected basic reproduction number. Confidence intervals were built around these estimates using the standard error formula developed by Becker and a similar subtraction of the number currently ill to calculate the modified standard error. The model was run using R 2.1.3.

\section{Results}

The original approach yielded estimates that were not statistically significantly different from the basic reproduction numbers selected for the model from 1 to 4 . However, estimates were significantly lower for the model runs with basic reproduction numbers of 4.5 and 5. The modified approach yielded estimates for the basic reproduction number that were not significantly different from any of the basic reproduction numbers from the original approach, though estimates were significantly lower than those selected for the model for the selected basic reproduction numbers of 4.5 and 5 .

\section{Conclusions}

The modified approach appears to yield valid estimates of the basic reproduction number within a reasonable margin of error, and performs at least as well as the original approach derived by Becker. Both approaches are vulnerable to the same instability when the case count is low or approaches the size of the at-risk population (as shown in the results for the original approach when applied at higher basic reproduction numbers), but this is less likely to be an issue when taking estimates toward the middle of an outbreak as in the modified approach instead of at the end.

\section{Keywords}

Mathematical Modeling; Transmission Dynamics; Basic Reproduction Number

\section{References}

1. Vynnycky E, White R. An Introduction to Infectious Disease Modelling. OUP Oxford. 2010 May 13.

2. Althaus C. Estimating the Reproduction Number of Ebola Virus (EBOV) During the 2014 Outbreak in West Africa. PLoS Curr. 2014 September 2; 6 .

3. Davoudi B, Miller J, Meza R, Meyers L, Earn D, Pourbohloul B. Early Real-Time Estimation of the Basic Reproduction Number of Emerging Infectious Diseases. Phys Rev X. 2012; 2(3).

4. Becker NG. Analysis of Infectious Disease Data. Chapman and Hall. 1989

5. Mossong J, Muller CP. Estimation of the Basic Reproduction Number of Measles During an Outbreak in a Partially Vaccinated Population. Epidemiol Infect. 2000; 124.

\section{${ }^{*}$ Carl Grafe}

E-mail: cgrafe@slco.org 